\title{
Preparation and Evaluation of Gastroretentive Floating Pellets of Metronidazole
}

\author{
Shahriar Nowshad and Md. Saiful Islam Pathan \\ Department of Pharmacy, State University of Bangladesh, Dhanmondi, Dhaka-1209, Bangladesh
}

\begin{abstract}
Traditionally metronidazole is used in the treatment of bacterial vaginosis, trichomoniasis, amoebiasis and is also used in combination with other drugs to treat Helicobacter pylori (H. pylori) that causes stomach or intestinal ulcers. The main aim of this study was to develop a gastroretentive floating pellets for the treatment of above pathological conditions. Such kind of dosage form may provide an extension of drug presence in the upper GI tract resulting enhanced absorption and improved bioavailability for the treatment against protozoa and bacteria. Nine formulations of metronidazole floating pellets such as F1, F2, F3, F4, F5, F6, F7, F8 and F9 were prepared by ionic gelation method using different quantities of sodium alginate and psyllium husk. The drug and polymer ratio were 1:1.4, $1: 1.5,1: 1.6,1: 1.6 ; 1: 1.7,1: 1.8,1: 1.8,1: 1.9$ and 1:2.0, respectively. The in vitro drug release study was carried out in $900 \mathrm{ml}$ phosphate buffer $(\mathrm{pH} 7.4)$ at $37 \pm 0.5^{\circ} \mathrm{C}$ and $50 \mathrm{rpm}$ for 12 hours using USP XXIV paddle method and the content of drug release was determined by UV spectrometer at $277 \mathrm{~nm}$. Maximum and minimum drug release were found in F1 (88.63\%) and in F6 (73.21\%), respectively. It indicates that increase in sodium alginate concentration decreases drug release. All the formulations were buoyant for more than 12 hours in simulated gastric fluid at $37^{\circ} \mathrm{C}$. The maximum and minimum bulk density tapped were 0.57 and 0.52 in F1 and F9, respectively where drug loading were $14.07 \%$ in $\mathrm{F} 1$ and $12.56 \%$ in F9 which indicates that bulk density of the pellets is directly proportional to drug loading. The maximum and minimum swelling were in F3 (75\%) and F7 (59\%), respectively which demonstrate that swelling of pellets were inversely proportional to the sodium alginate concentration but it is directly proportional to psyllium husk content. In addition, the psyllium husk keeps the GI tract healthy by scavenging toxins and residues of digestive systems. Therefore, it can be concluded that combination of sodium alginate and biodegradable psyllium husk can be prospectively used for the preparation of gastroretentive floating pellets.
\end{abstract}

Key words: Gastric retension, floating pellet, ionotropic gelatination technique, metronidazole

\section{Introduction}

The design of oral control drug delivery systems (DDS) is primarily aimed to achieve more predictable and increased bioavailability. Now-a-days most of the pharmaceutical scientists are involved in developing the ideal DDS, which should have advantage of single dose for the whole duration of treatment and it should deliver the active drug directly at the specific site. Scientists have succeeded to develop a system and it encourages the scientists to develop control release systems. Control release implies the predictability and reproducibility to control the drug release, drug concentration in target tissue and optimization of the therapeutic effect of a drug by modifying its release in the body with lower and less frequent doses.
Under certain circumstances prolonging the gastric retention of a delivery system is desirable for achieving greater therapeutic benefit of the drug substances. For example, drugs that are absorbed in the proximal part of the gastrointestinal tract and the drugs that are less soluble or are degraded by the alkaline $\mathrm{pH}$ may benefit from the prolonged gastric retention. In addition, for local and sustained drug delivery to the stomach and the proximal small intestine to treat certain conditions, prolonging gastric retention of the therapeutic moiety may offer numerous advantages including improved bioavailability, therapeutic efficacy and possible reduction of the dose size. The main principle of GRDDS is to prolong the release of drug at the site of absorption and one of the most recent approaches for the prolongation of gastric retention time is the floating drug delivery systems. Due to

Correspondence to: Md. Saiful Islam Pathan, E-mail: <sip@sub.edu.bd> 
low bulk density of the floating dosages form, they can float in the gastric environment for longer period of time which attributes to improve bioavailability of the drug (Nayak et al., 2010).

It is known that gastroretention time of a drug is affected by several factors, but the gastric emptying time in human is particularly influenced by the size of the orally administered object. Therefore, various approaches for prolonging drug gastroretention have been investigated and floating system has been found to be promising as it prolongs the gastroretention without influencing the size of the orally administered dosage form. Thus, metronidazole will be very effective for the treatment of H. pylori infection. At the same time it will be very much effective in the treatment of other antibacterial and antiprotozoal deseases. Hence, the present study was designed to develop floating pellets of metronidazole using sodium alginate and biodegradable natural polymer psyllium husk (Biswas et al., 2012)

\section{Materials and Methods}

Metronidazole was obtained as a gift sample from Globe Pharmaceuticals Ltd., Noakhali. Sodium alginate and calcium chloride were purchased from Loba Chemie, India. Psyllium husk was procured from Sajib limited, Bangladesh. All other chemicals used were of analytical grade.

Method of preparation of Pellets: Pellets were prepared by ionotropic gelatination technique. To prepare metronidazole floating pellets sodium alginate and psyllium husk were accurately weighed and dissolved in small amount of distilled water and soaked for overnight. Then psyllium husk was filtered and added to sodium alginate. The mixture was homogenized using propeller stirrer (2775 rpm) for 30 minutes. Metronidazole $(500 \mathrm{mg})$ and distilled water were added to make final volume up to $35 \mathrm{ml}$., and was homogenized thoroughly using propeller stirrer for 30 minutes to form smooth viscous dispersion. Then liquid paraffin was added to the above viscous dispersion and homogenized for 15 minutes using propeller stirrer and then sprayed on to cationic solution $\left(\mathrm{CaCl}_{2}, 4 \%\right)$ using a syringe having diameter $21 \mathrm{G}$ from a height of $4 \mathrm{~cm}$. Sprayed droplets were retained for 2-3 minutes to complete curing reaction. The Pellets were collected by decantation and washed repeatedly by distilled water, dried at room temperature for 12 hours (Biswas et al., 2012)

Particle size analysis: The diameter of beads was determined by using micrometer. For this purpose, 10 dried pellets were randomly selected from each batch and diameter was measured from three different angles of each pellets. The mean diameters were calculated using following formula. Mean particle size $=\sum$ (mean particle size of the fraction $\times$ weight fraction) $/ \sum$ (weight fraction) (Chourasia et al, 2004). Color and shape of dried beads of each batch were observed under trinocular microscope and light microscope.

Surface morphology: The surfaces and cross-section morphologies of the pellets were observed with a scanning electron microscope (SEM) (JSM-6490 LA, JEOL, Tokyo, Japan) operated at an acceleration voltage of $25 \mathrm{kV}$. The pellets were made conductive by sputtering thin coat of platinum under vaccum using Jeol JFC-1600 autofine coater and then the images were recorded at different magnifications.

Determination of drug content and entrapment efficiency: The pellets (100mg) were weighed and crushed in a mortar and pestle and the crushed material was dissolved in $10 \mathrm{ml}$ of phosphate buffer. The mixture was shaken for about 15 minutes. The solution was then made up to $100 \mathrm{ml}$ with phosphate buffer. From this solution 1 $\mathrm{ml}$ was taken into $100 \mathrm{ml}$ volumetric flask and $99 \mathrm{ml}$ of phosphate buffer (pH7.4) was added for dilution. Metronidazole content was estimated by UVspectrophotometer at $277 \mathrm{~nm}$. The drug content and entrapment efficiency were determined by the following equations (Nappinnai et al., 2007).

$\%$ Drug content $=\{$ Weight of drug present in pellets $(\mathrm{gm}) /$ Weight of quantity of pellets $(\mathrm{gm})\} \times 100$

$\%$ Entrapment efficiency $=($ Experimental drug content/Theoretical drug content) $\times 100$

FTIR studies: The infrared (IR) spectra were recorded using an FTIR spectrophotometer (Shimadzu FTIR $8400 \mathrm{~S}$, Japan) by the $\mathrm{KBr}$ pellet method between 4000 and $400 \mathrm{~cm}^{-1}$. The spectra obtained for metronidazole and physical mixtures of metronidazole with polymers were compared to check compatibility of drug with polymers.

In vitro buoyancy study: The time between the introduction of the pellets into the medium and its 
buoyancy to the upper one third of the dissolution vessel (floating lag time) and the time for which the formulation constantly floated on the surface of the medium (floating duration) were measured simultaneously. The $100 \mathrm{mg}$ of pellets were placed in a beaker filled with $50 \mathrm{ml} 0.1 \mathrm{~N}$ hydrochloric acid. Temperature was maintained at $37^{\circ} \mathrm{C}$. The floating time of pellets was observed for $12 \mathrm{hrs}$ and floating time was determined (Sharma et al., 2006).

Determination of bulk density: At first all the pellets were taken in a $10 \mathrm{ml}$ measuring cylinder and their volume was noted. This is called untapped volume. Then tapped for 100 times and the volume was again noted. This is known as tapped volume.

Bulk density, untapped $=$ weight of all pellets (gm)/untapped volume (ml)

Bulk density, tapped $=$ weight of all pellets (gm)/tapped volume (ml)

Determination of swelling index: Pellets (100 mg) were placed in a little excess of distilled water, $0.1 \mathrm{~N} \mathrm{HCl}$ and phosphate buffer ( $\mathrm{pH}$ 7.4) and allowed to swell to constant weight. The pellets were removed, blotted with filter paper and their changes in weight were measured at an interval of 10 minutes and recorded. This process was repeated until equilibrium was obtained (Mazumderet al, 2010). The degree of swelling was calculated from the following formula:

Swelling index $=$ (Final wt. of pellets - Initial wt. of pellets) / Initial wt. of pellets $\times 100$

Drug release studies: For this, $20 \mathrm{mg}$ equivalent metronidazole loaded sodium alginate pellets from each formulation were placed in the vessel of USP Type II dissolution apparatus containing $900 \mathrm{ml}$ of phosphate buffer ( $\mathrm{pH}$ 7.4) maintained at $37 \pm 0.5^{\circ} \mathrm{C}$ and stirred at 50 rpm. $10 \mathrm{ml}$ of samples were collected periodically (every 60 minutes) and replaced with a fresh dissolution medium, phosphate buffer ( $\mathrm{pH}$ 7.4). These samples were filtered with filter paper and analyzed for the drug present in them with the help of a UV spectrophotometer at $277 \mathrm{~nm}$ against phosphate buffer ( $\mathrm{pH}$ 7.4) as blank. Only those batches were selected for the release study, which have good drug content and drug entrapment efficiency more than $50 \%$.

Study of drug release kinetics: The in vitro data were treated according to zero order, first order, Higuici, Korsmeyer-Peppas, Hixson-Crowell equations and the coefficient of co-relation were determined (Jain et al., 2005)

Zero order equation $-\%$ release $=$ K.time

First order equation $-\log$ (fraction unreleased) $=$ K/2.303.time

Higuici equation $-\%$ release $=$ K.time ${ }^{0.5}$

Korsmeyer-Peppas equation - \% released $=K \cdot$ time $^{\mathrm{n}}$

Hixson-Crowell equation $-(\text { fraction of unreleased })^{1 / 3}=1$ K.time

\section{Results and Discussion}

Evaluation of pellets preparation method: In this study, gastroretentive floating metronidazole pellets were prepared using Na-alginate and biodegradable psyllium husk as polymer using ionic gelation technique. The composition of all formulations are described in Table 1.

Size and morphology studies: Mean surface diameter of gastroretentive floating pellets of metronidazole were between $1.021 \pm 0.0732$ to $1.027 \pm 0.0381$ (Figure 1). The pellets are spherical, free flowing and discrete. The bulk densities were between 0.50 to 0.0 .56 (untapped) \& 0.52 to 0.57 (tapped) (Table 2).

Table 1. Formulations of gastroretentive floating pellets of metronidazole.

\begin{tabular}{llllllllll}
\hline Ingredients & \multicolumn{8}{c}{ Formulation Code } \\
\cline { 2 - 9 } & F1 & F2 & F3 & F4 & F5 & F6 & F7 & F8 & F9 \\
\hline Metronidazole (mg) & 500 & 500 & 500 & 500 & 500 & 500 & 500 & 500 & 500 \\
Na-alginate (mg) & 405 & 455 & 505 & 405 & 455 & 505 & 405 & 455 & 505 \\
Psyllium Husk (mg) & 300 & 300 & 300 & 400 & 400 & 400 & 500 & 500 & 500 \\
Ratio(D: P) & $1: 1.4$ & $1: 1.5$ & $1: 1.6$ & $1: 1.6$ & $1: 1.7$ & $1: 1.8$ & $1: 1.8$ & $1: 1.9$ & $1: 2.0$ \\
Distilled water (ml) & 35 & 35 & 35 & 35 & 35 & 35 & 35 & 35 & 35 \\
Liquid paraffin (ml) & 2.75 & 2.75 & 2.75 & 2.75 & 2.75 & 2.75 & 2.75 & 2.75 & 2.75 \\
\hline
\end{tabular}


Table 2. Physical properties and percentage yield of metronidazole pellets.

\begin{tabular}{lccccc}
\hline Formulation & \% Yield & \multicolumn{2}{c}{ Bulk density $(\mathrm{gm} / \mathrm{ml})$} & \multirow{2}{*}{$\begin{array}{c}\text { Mean } \\
\text { Diameter } \pm \text { RSD }\end{array}$} & Shape of beads \\
\cline { 3 - 4 } & & Untapped & Tapped & $1.027 \pm 0.0381$ & Spherical \\
\hline F1 & 83 & 0.56 & 0.57 & $1.026 \pm 0.0734$ & Spherical \\
F2 & 92 & 0.55 & 0.54 & $1.024 \pm 0.0231$ & Spherical \\
F3 & 92 & 0.54 & 0.55 & $1.022 \pm 0.0557$ & Spherical \\
F4 & 92 & 0.52 & 0.54 & $1.026 \pm 0.0312$ & Spherical \\
F5 & 88 & 0.51 & 0.52 & $1.023 \pm 0.0921$ & Spherical \\
F6 & 96 & 0.52 & 0.53 & $1.022 \pm 0.0545$ & Spherical \\
F7 & 82 & 0.51 & 0.52 & $1.023 \pm 0.0154$ & Spherical \\
F8 & 88 & 0.52 & 0.53 & $1.021 \pm 0.0732$ & Spherical \\
F9 & 87 & 0.50 & 0.52 & & \\
\hline
\end{tabular}

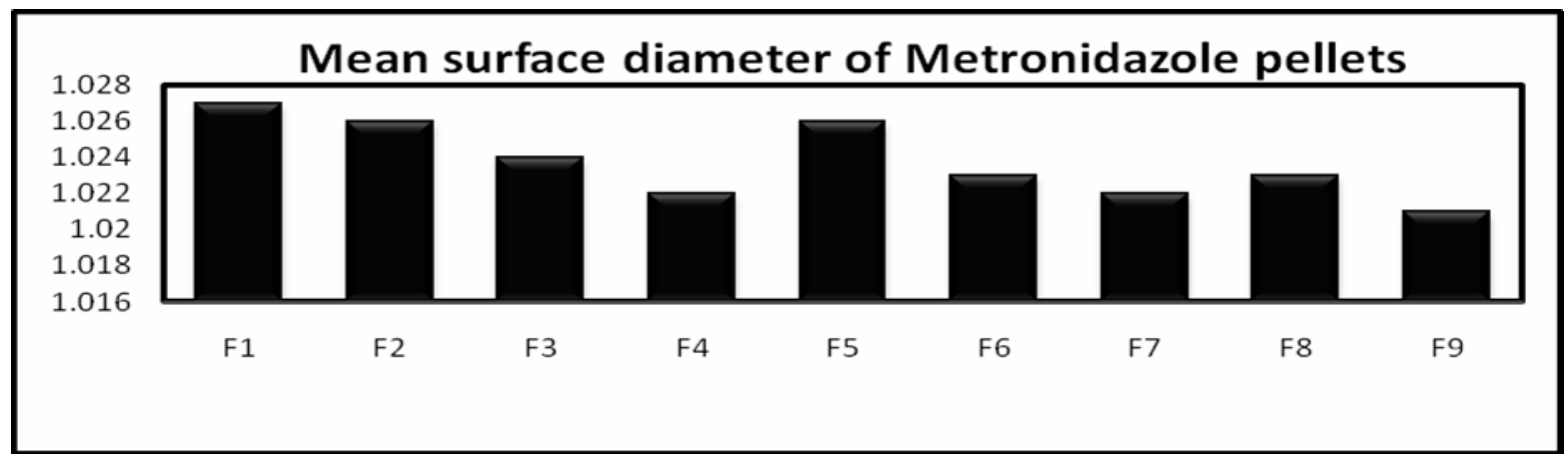

Figure 1. Mean surface diameter of metronidazole floating pellets

Surface morphology: SEM was used to observe the surface morphology of gastroretentive floating pellets of metronidazole. It was observed that the surface was rough (Figure 2) and further magnification (Figures 3 and 4) some particles were found. It indicates that the drug was successfully incorporated in the pellets.

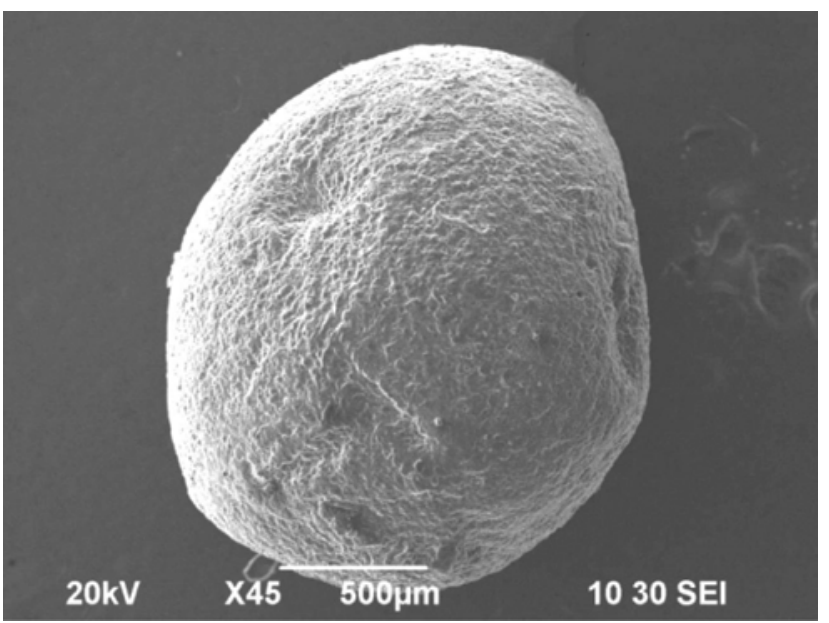

Figure 2. SEM of metronidazole pellets.

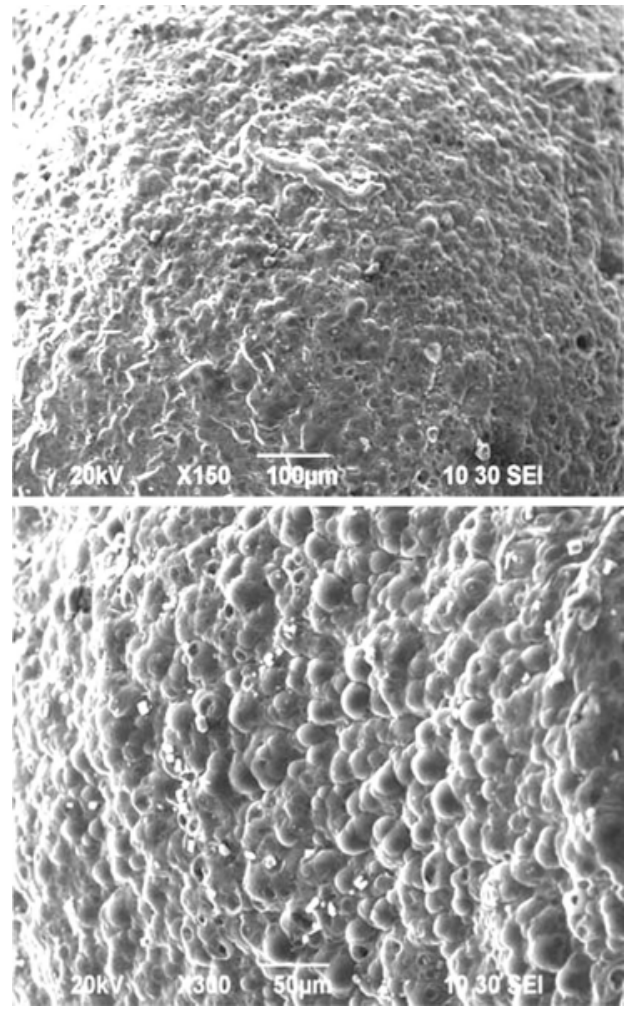

Figures 3 and 4: SEM of metronidazole pellets (magnified) 
Drug content and entrapment efficiency: The drug content and entrapment efficiency of metronidazole floating pellets were between $14.07 \%$ to $12.56 \%$ and $99 \%$ to $94 \%$ respectively (Table 3), Figure 5). The drug content of metronidazole floating pellets were inversely proportional to polymer content which may be due to enhanced viscosity of the combination polymer during entrapment process.

Here the bulk density in F1 was found to be maximum of $0.56 \mathrm{gm} / \mathrm{ml}$ (untapped) and $0.57 \mathrm{gm} / \mathrm{ml}$ (tapped) and the minimum in F9 $0.50 \mathrm{~g} / \mathrm{ml}$ (untapped) and $0.52 \mathrm{~g} / \mathrm{ml}$ (tapped). These results indicate that the bulk density of the pellets is directly proportional to drug loading.
Floating test of gastroretentive floating pellets of metronidazole: The floating test of metronidazole floating pellets was performed in $0.1 \mathrm{~N} \mathrm{HCl}$. The time has been shown in table 4.

All the pellets show good floating characteristics. There was no floating lag time for any of the formulations. Floating time was found to be more than 12 hours.

FTIR studies: The FT-IR spectra analysis of metronidazole and physical mixtures shows that there was no significant interaction between drug and polymers as shown in figure- 7, 8. It indicates that polymers were compatible with metronidazole.

Table 3. Percentage drug content and entrapment efficiency of metronidazole floating pellets.

\begin{tabular}{cccc}
\hline Formulation & Theoretical drug content (\%) & Drug content (\%) & Entrapment efficiency (\%) \\
F1 & 14.32 & 14.07 & 98 \\
F2 & 14.12 & 14.05 & 99 \\
F3 & 13.93 & 13.27 & 95 \\
F4 & 13.93 & 13.26 & 95 \\
F5 & 13.71 & 13.06 & 95 \\
F6 & 13.56 & 12.84 & 95 \\
F7 & 13.56 & 12.70 & 94 \\
F8 & 13.37 & 12.63 & 94 \\
F9 & 13.21 & 12.56 & 95 \\
\hline
\end{tabular}

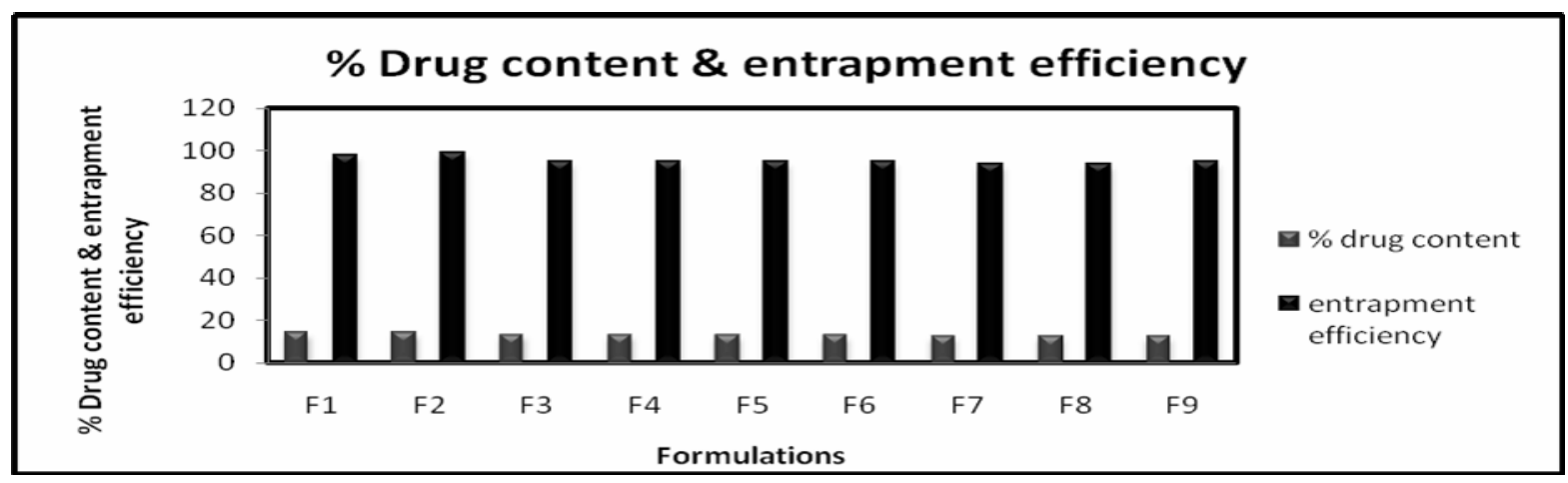

Figure 5. \% Drug content and entrapment efficiency of metronidazole floating pellet.

Table 4. Floating time of different formulations.

\begin{tabular}{lcccccccccc}
\hline Test & F1 & F2 & F3 & F4 & F5 & F6 & F7 & F8 & F9 \\
\hline Floating time & $>12 \mathrm{hrs}$ & $>12 \mathrm{hrs}$ & $>12 \mathrm{hrs}$ & $>12 \mathrm{hrs}$ & $>12 \mathrm{hrs}$ & $>12 \mathrm{hrs}$ & $>12 \mathrm{hrs}$ & $>12 \mathrm{hrs}$ & $>12 \mathrm{hrs}$ \\
\hline
\end{tabular}




\section{Drug content \& bulk density relationship}

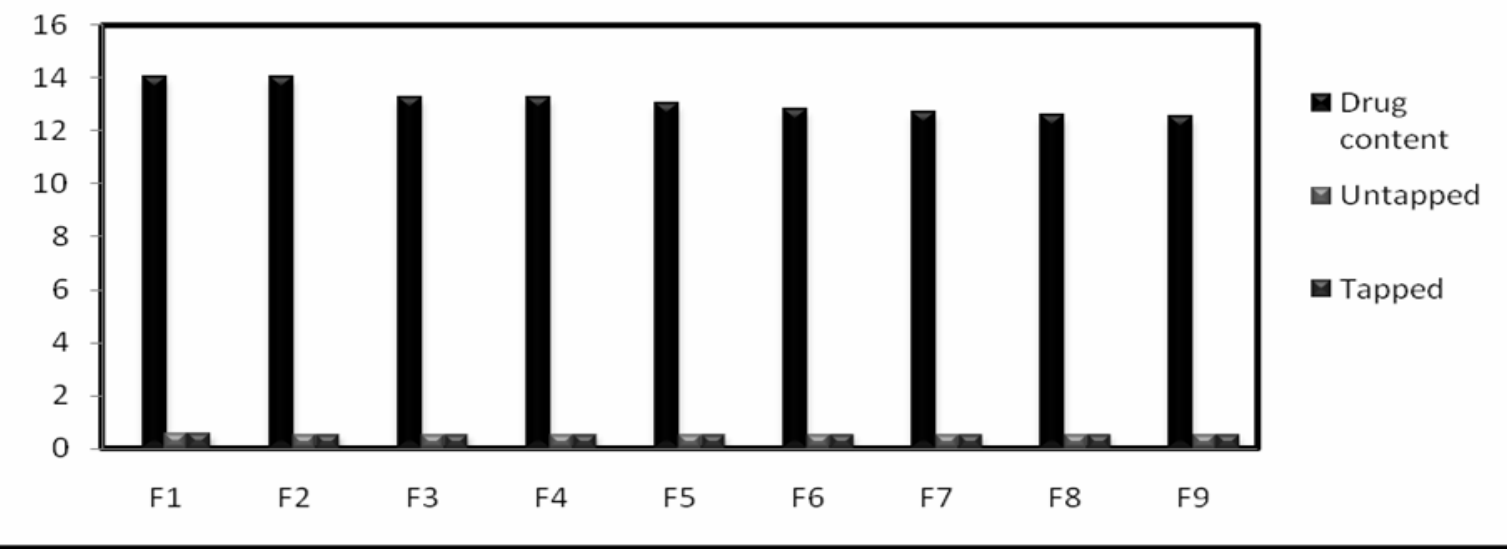

Figure 6: \% Drug content and bulk density of metronidazole floating pellet.

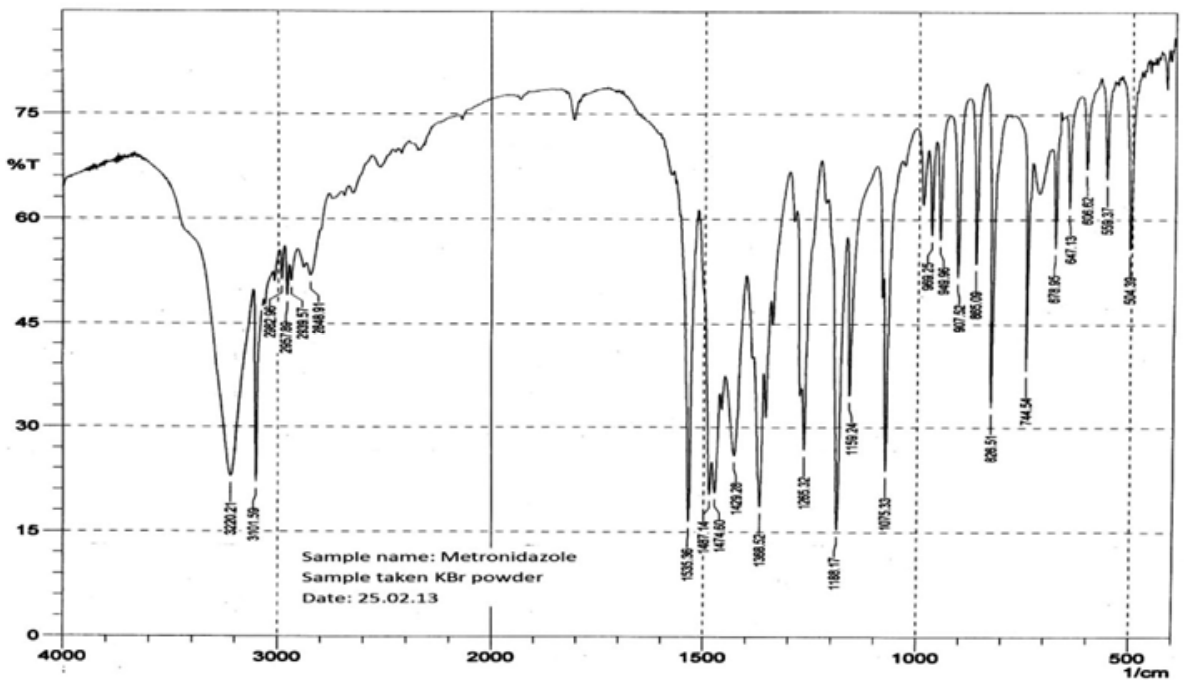

Figure 7. FTIR spectra of metronidazole

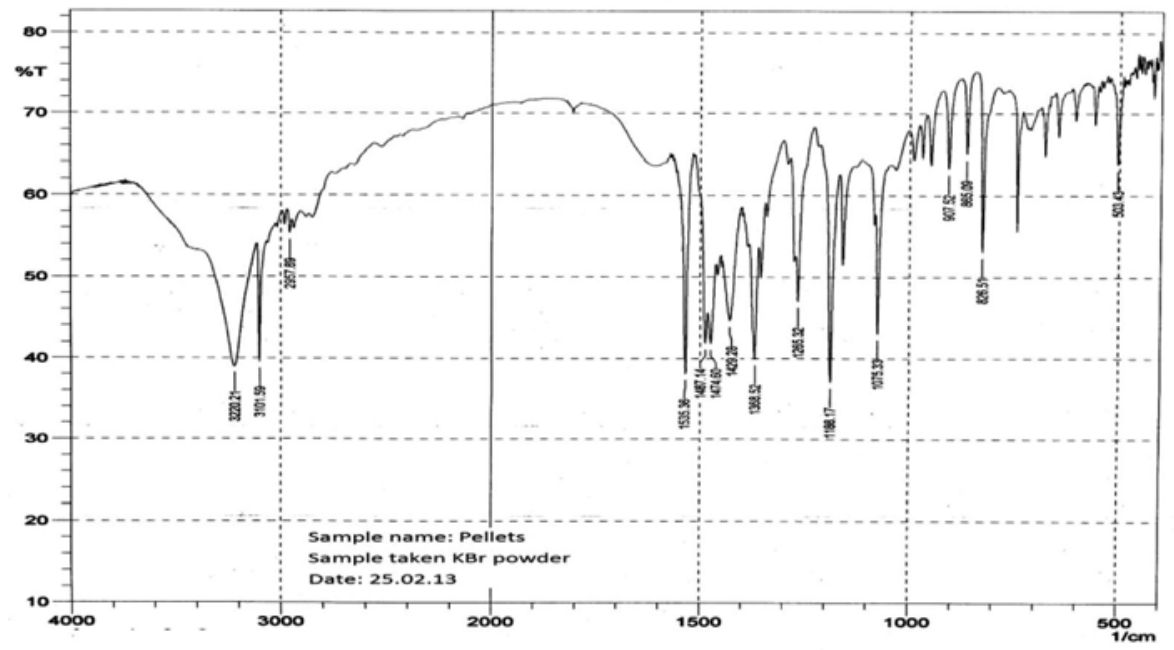

Figure 8. FTIR spectra of metronidazole pellets with sodium alginate and psyllium husk 
Swelling index of metronidazole floating pellets: The pellets were significantly swelled in phosphate buffer having $\mathrm{pH} 7.4$, than any other media. The swelling indices of pellets were inversely proportional to the sodium alginate content, but directly proportional to psyllium husk content (Table 5, Figure 9)

In vitro drug release study: The in vitro drug release study was carried out in $900 \mathrm{ml}$ phosphate buffer (pH 7.4) at $37 \pm 0.5^{\circ} \mathrm{C}$ and $50 \mathrm{rpm}$ for 12 hours using USP XXIV paddle method and the content of drug release was determined by UV spectrometer at $277 \mathrm{~nm}$. Drug release were found in F1 (88.63\%), F2 (82.62\%) and F3 $(79.84 \%)$. It indicates that increase in sodium alginate concentration decreases drug release (Figure10). Drug release were found in F4 (79.39\%), F5 (77.25\%) and F6 (73.21\%). It indicates that increase in sodium alginate concentration decreases drug release (Figure11). Drug release were found in F7 (84.27\%), F8 (82.22\%) and F9 $(81.81 \%)$. It indicates that increase in sodium alginate concentration decreases drug release (Figure 12).

\section{Table 5. Percentage of swelling index.}

\begin{tabular}{llccccccccc}
\hline SI. No. & Solvent & F1 (\%) & F2 (\%) & F3 (\%) & F4 (\%) & F5 (\%) & F6 (\%) & F7 (\%) & F8 (\%) & F9 (\%) \\
\hline 01 & Distilled $\mathrm{H}_{2} \mathrm{O}$ & 42 & 34 & 33 & 48 & 45 & 44 & 49 & 43 & 42 \\
02 & Buffer (pH7.4) & 66 & 62 & 59 & 72 & 69 & 67 & 75 & 72 & 71 \\
03 & $0.1 \mathrm{~N} \mathrm{HCl}$ & 33 & 31 & 28 & 37 & 36 & 33 & 41 & 38 & 37 \\
\hline
\end{tabular}

\section{Swelling indices of metronidazole floating pellets}

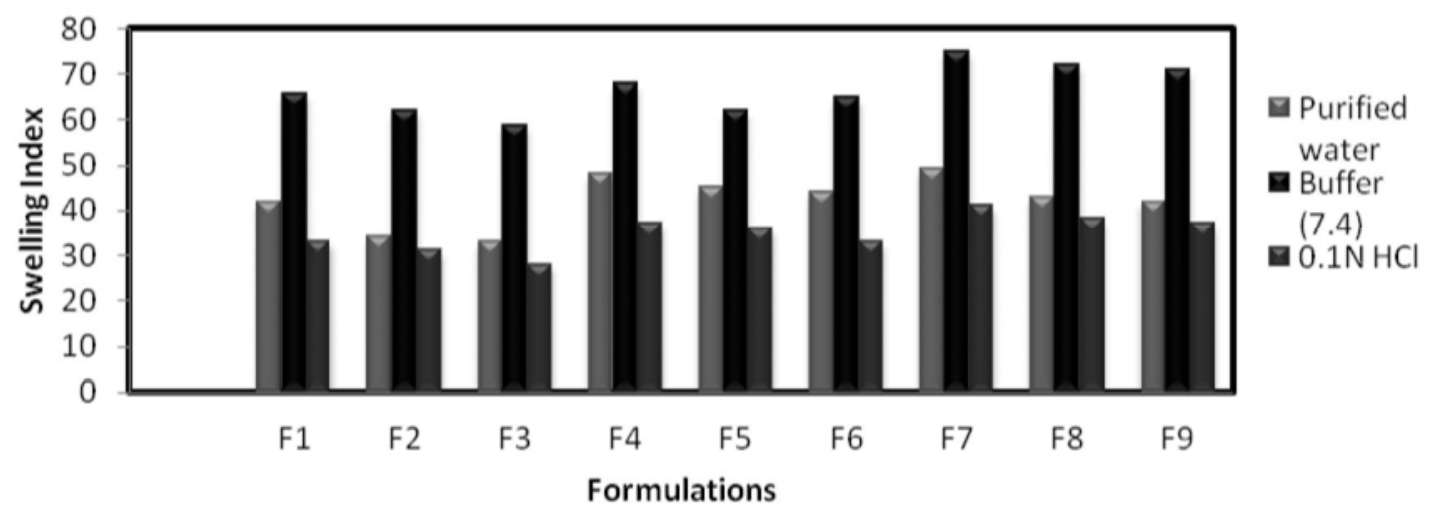

Figure 9. Swelling indices of metronidazole floating pellets.

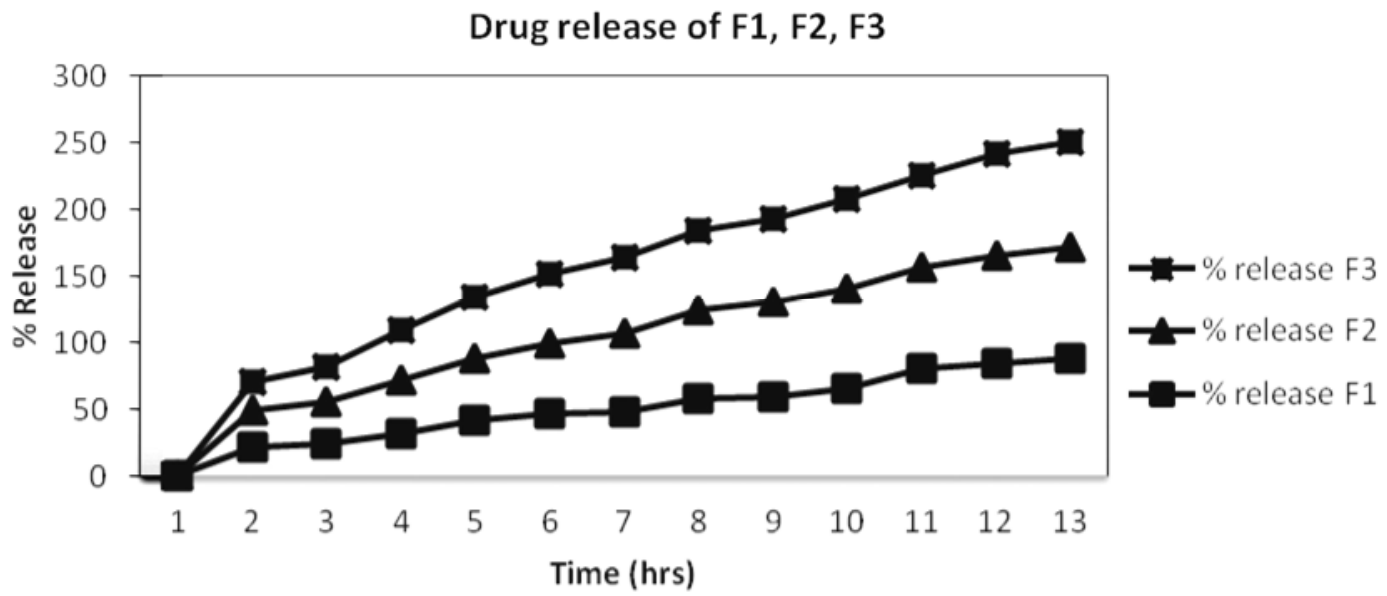

Figure 10. Drug release study of formulation F1, F2 and F3. 


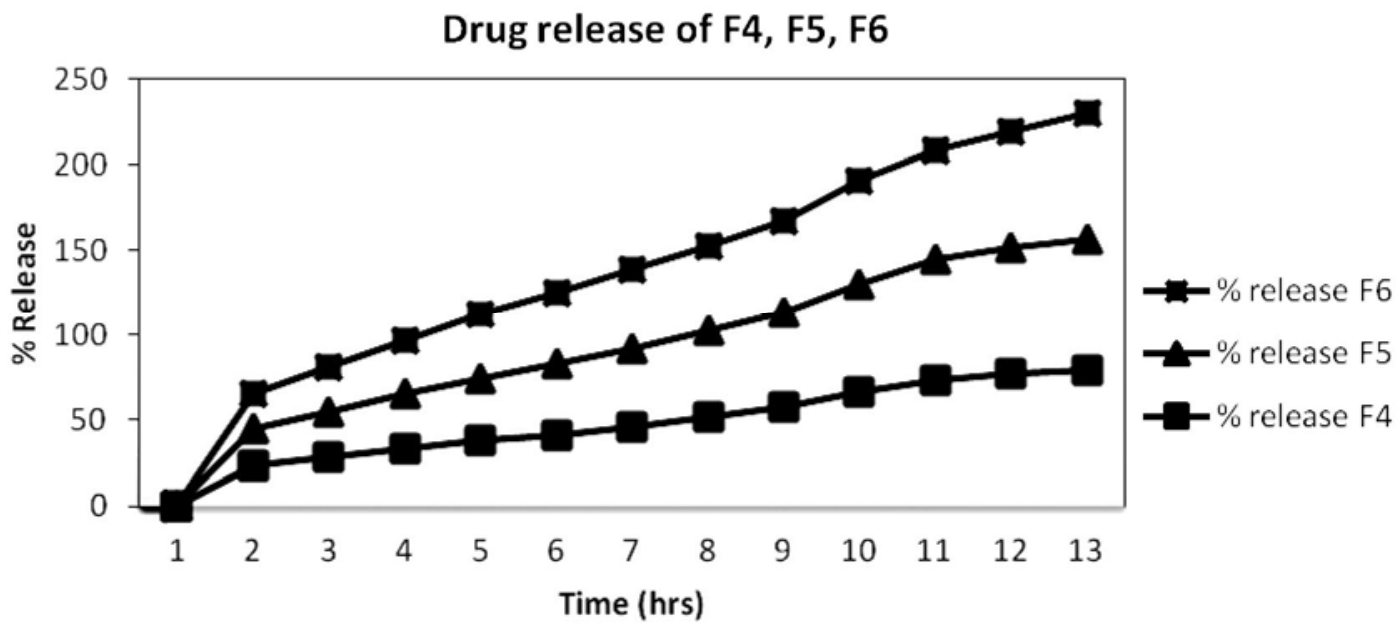

Figure 11. Drug release study of F4, F5 and F6.

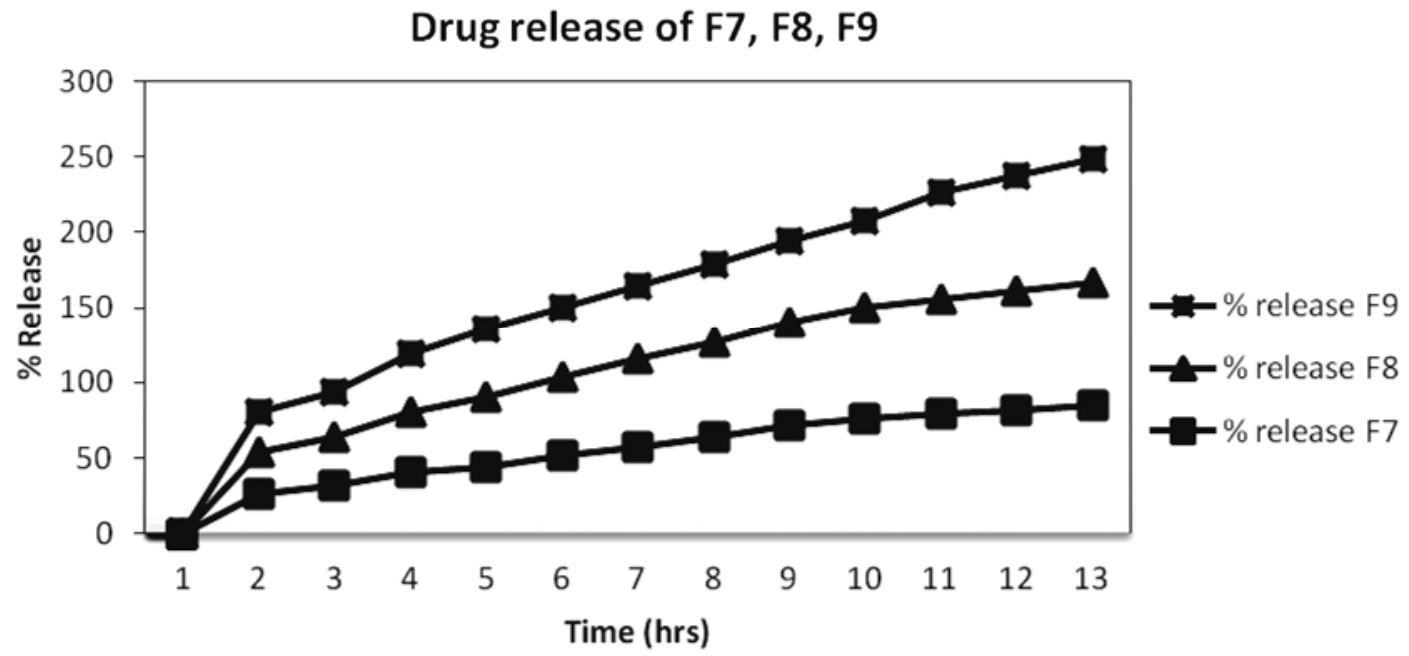

Figure 12. Drug release study of F7, F8 and F9.

Table 6. Model fitting release profile of metronidazole floating pellets.

\begin{tabular}{lllllll}
\hline Formulation & $\begin{array}{l}\text { Zero- } \\
\text { order } r^{2}\end{array}$ & $\begin{array}{l}\text { First-order } \\
\mathrm{r}^{2}\end{array}$ & $\begin{array}{l}\text { Higuchi } \\
\text { matrix } \mathrm{r}^{2}\end{array}$ & $\begin{array}{l}\text { Korsmeyer- } \\
\text { Peppas } \mathrm{r}^{2}\end{array}$ & $\begin{array}{l}\text { Hixson- } \\
\text { Crowell } \mathrm{r}^{2}\end{array}$ & $\begin{array}{l}\text { Best Fitted } \\
\text { F1 }\end{array}$ \\
0.973 & 0.925 & 0.958 & 0.952 & 0.960 & Zero- order \\
F2 & 0.927 & 0.991 & 0.992 & 0.975 & 0.981 & Higuchi-matrix \\
F3 & 0.926 & 0.975 & 0.989 & 0.980 & 0.972 & Higuchi-matrix \\
F4 & 0.965 & 0.960 & 0.963 & 0.947 & 0.974 & Hixon-Crowell \\
F5 & 0.969 & 0.969 & 0.970 & 0.965 & 0.980 & Hixon-Crowell \\
F6 & 0.950 & 0.980 & 0.988 & 0.984 & 0.979 & Higuchi-matrix \\
F7 & 0.940 & 0.990 & 0.991 & 0.980 & 0.987 & Higuchi-matrix \\
F8 & 0.928 & 0.993 & 0.995 & 0.981 & 0.985 & Higuchi-matrix \\
F9 & 0.929 & 0.909 & 0.991 & 0.931 & 0.929 & Higuchi-matrix \\
\hline
\end{tabular}


In vitro dissolution kinetics of metronidazole floating pellets: To analyze mechanism of drug release and release rate kinetics from the metronidazole floating pellets, the data obtained were fitted in zero-order release, first-order kinetic, Higuichi-matrix, Korsmeyer-Peppas and HixsonCrowell equation (Table 6).

Formulations F1, F2 and F3 containing drug- polymer ratio of $1: 1.41,1: 1.51,1: 1.61$ and percent drug release after 12 hours were $88.63,82.62$ and $79.84 \%$, respectively. Formulations F4, F5 and F6 containing drugpolymer ratio of 1:1.61, 1:1.71, 1:1.81 and percent drug release after 12 hours were $79.39,77.25$ and $73.21 \%$, respectively. Formulations F7, F8 and F9 containing drugpolymer ratio $1: 1.81,1: 1.91,1: 2.0$ and percent drug release after 12 hours were $84.27,82.22$ and $81.81 \%$, respectively.

It is quite evident from the above that the drug release decreases as the amount of sodium alginate increases. In this model curve most of formulations are best fitted to Higuchi-matrix model.

\section{Conclusions}

In the present study metronidazole gastroretentive floating pellets were successfully prepared using sodium alginate and psyllium husk to increase gastric retention time and subsequently to retard drug release. From the above experiment we can conclude that:

$>$ Psyllium husk and sodium alginate can be used as combination polymer to make gastroretentive floating pellets

$>$ Sodium alginate decreases the drug release with the increase in its concentration.

$>$ Bulk density of the pellets is directly proportional to drug loading.

$>$ Sodium alginate decreases the swelling of pellets with the increase in its concentration but psyllium husk increases the swelling of pellets with the increase in its concentration.
$>$ In addition to the above psyllium husk is a natural biodegradable polymer which helps to keep the GI tract healthy by soaking up toxins and harmful residues of digestive systems.

> Further study on formulation optimization, stability and in-vivo study will confirm the appropriateness of these formulated gastroretentive metronidazole floating pellets.

\section{Acknowledgements}

The authors wish to thank the Globe Pharmaceuticals Limited, Noakhali for providing gift sample of Metronidazole.

\section{References}

Nayak, Amit Kumar., Maji Ruma, Das Biswarup.2010.

Gastroretentive drug delivery systems: a review. Asian $J$. Pharm.Sci.Res.3. 2-10

Biswas, S.K., Paul, S. Chowdhury A. and Das J. 2012. Preparation and evaluation of gastroretentive floating pellets of metronidazole using Na-alginate and hydroxyl propyl methyl cellulose polymers. Pakistan J. Biol. Sci. 15, 290-295.

Chourasia, M.K. and Jain, S.K. 2004. Potential of guar gum microspheres for target specific drug release to colon. $J$. Drug Targeting 12, 435-442.

Nappinnai, M. and Kishore, V.S. 2007. Formulation and evaluation of microspheres of diltiazemhydrochloride. I. J. Pharm. Sci. 69, 511-514.

Sharma, S. and Pawar, A. 2006. Low density multiparticulate system for pulsatile release of meloxicam. Int J Pharm, 313,150-158.

Mazumder Rana, Nath Lila K, Haque Anwarul, Maity Tarasankar, Chowdhury Prasanta K, Shrestha Bhupendra, Chakraborty Manas and Pal Rabindra N 2010. Formulation and in vitro evaluation of natural polymers based microspherses for colonic drug delivery. Int. J Pharmac. Pharm. Sci. 2, 211-219

Jain, S.K, Awasthi, Am., Jain, N.K and Agarwal, G.P. 2005. Calcium silicate based microspheres of repaglinide for gastroretentive floating drug delivery: Preparation and in vitro characterization. J. Control. Release, 107, 300-309. 